\title{
IC/Microfluidic Hybrid System for Magnetic Manipulation of Biological Cells
}

\author{
Hakho Lee, Yong Liu, Student Member, IEEE, Robert M. Westervelt, and Donhee Ham, Member, IEEE
}

\begin{abstract}
This paper introduces an integrated circuit (IC)/microfluidic hybrid system for magnetic manipulation of biological cells. The hybrid system consists of an IC and a microfluidic system fabricated on top. Biological cells attached to magnetic beads are suspended inside the microfluidic system that maintains biocompatibility. The IC contains a microcoil array circuit that produces spatially-patterned microscopic magnetic fields. Programmable, rapid reconfiguration of the field pattern made possible by the IC allows an efficient simultaneous manipulation of multiple individual bead-bound cells with precise position control. Two prototypes, SiGe/microfluidic and CMOS/microfluidic hybrid systems, validate the proposed approach.
\end{abstract}

Index Terms-Bioelectronics, biolab-on-IC, cell manipulations, CMOS integrated circuits, digital logic, integrated circuits, lab-on-a-chip, microcoil arrays, microcoils, microfluidics, SiGe integrated circuits.

\section{INTRODUCTION}

$\mathbf{T}$ ODAY'S semiconductor integrated circuits (ICs) can contain over 100 million transistors, operate at $\mathrm{GHz}$ speeds, process Gbyte data, and can be manufactured inexpensively. With these advantages, ICs have become one of the most significant enabling technologies of our time, lying at the heart of today's advanced computers, communication systems, and multimedia hardware.

There has lately been a growing interest in exploiting the benefits of the ICs for areas outside the traditional application spaces. One notable such area is found in biology. Bioanalytical instruments have been miniaturized on ICs to study neural activities and tissue dynamics, to monitor ion channels and electrochemical activities, to probe DNA, and to electrically manipulate cells [1]-[6]. These biolab-on-IC systems utilize the IC to facilitate faster, repeatable, and standardized biological experiments at low cost with a small volume of biological sample. The research activities on biolab-on-IC are expected to enjoy substantial growth in the foreseeable future. This trend is reflected by the increasing number of publications in major IC design

Manuscript received August 2, 2005; revised January 7, 2006. This work was supported by an IBM Faculty Partnership Award, NSF Grant ECS-0313143, NSF Grant PHY-0117795, and the NSF Nanoscale and Engineering Center (NSEC).

H. Lee was with the Department of Physics, Harvard University, Cambridge, MA 02138 USA. He is now with the MGH-Harvard Center for Molecular Imaging Research, Charlestown, MA 02129 USA (e-mail: hlee18@ partners.org).

Y. Liu and D. Ham are with Electrical Engineering, Division of Engineering and Applied Sciences (DEAS), Harvard University, Cambridge, MA 02138 USA (e-mail: yongliu@deas.harvard.edu; donhee@ deas.harvard.edu).

R. M. Westervelt is with the Department of Physics and DEAS, Harvard University, Cambridge, MA 02138 USA (e-mail: westervelt@deas.harvard.edu).

Digital Object Identifier 10.1109/JSSC.2006.874331

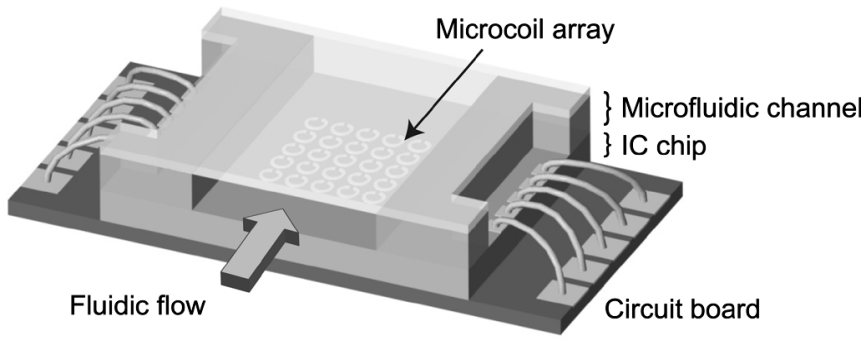

Fig. 1. Conceptual illustration of the IC/microfluidic hybrid system.

conferences and journals such as the IEEE International SolidState Circuits Conference and the IEEE JouRnAL OF SOLIDSTATE CiRCUITS, e.g., [2]-[6].

As a new biolab-on-IC, this paper introduces an IC/microfluidic hybrid system for magnetic manipulation of biological cells. The hybrid system consists of an IC and a microfluidic system placed on top as schematically illustrated in Fig. 1. A microcoil array circuit incorporated in the IC generates spatially-patterned microscopic magnetic fields to simultaneously control the motion of many individual cells (tagged by magnetic beads) suspended inside the microfluidic system. The programmability and speed of the microcoil array circuit enabled by the IC make the manipulation operation efficient and versatile. The microfluidic system provides a biocompatible environment.

The hybrid manipulation system combining the power of ICs and biocompatibility of microfluidics adds a new direction in the field of biolab-on-IC, offering possibilities for new types of investigations in biomedicine and systems biology [7]. For example, the hybrid system can be used to bring together a small number of individual cells with tight spatial control to study cell-cell interactions at the single cell level, which allows for explicit measurement of cell communications without averaging them [8]. For another example, the hybrid system can assemble a 2-D artificial biological tissue at the microscale, by bringing a large number of cells one by one into a desired geometry. The engineered tissue can be used to assay the development of a tissue from different types of cell populations [7], [9].

This paper describes the operation of the IC/microfluidic hybrid system using two prototypes we constructed. The first prototype utilizing a SiGe IC (Section III) demonstrates the basic concept with a rather simple operation of the IC. This specific prototype work was published in [10] and is presented here as a review. The new, second prototype, a CMOS/microfluidic hybrid system (Section IV), fully exploits the power of CMOS ICs for efficient and versatile cell manipulation. Before discussing the prototype details, we will first review the basic operating principle of the hybrid system in Section II. 
(a)

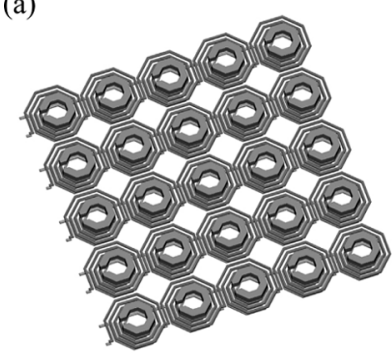

(b)

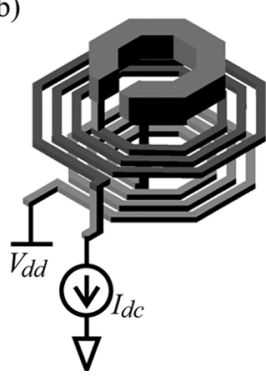

Fig. 2. (a) Microcoil array. (b) Microcoil with a current source.

\section{IC/MiCROFLUIDIC HYBRID SYSTEM}

\section{A. Overview}

The hybrid system consists of an IC and a microfluidic system fabricated on top as conceptually illustrated in Fig. 1. Biological cells attached to magnetic beads are suspended inside the microfluidic system where biocompatibility is maintained. The IC contains an array of microcoils [Fig. 2(a) shows a microcoil array example], which produces spatially-patterned microscopic magnetic fields on the surface of the IC. In a given magnetic field pattern, the bead-bound cells are attracted toward local field magnitude peak positions and become trapped there. ${ }^{1}$ Therefore, by reconfiguring the spatial field pattern and hence by moving the field magnitude peak positions, the individual bead-bound cells can be transported to their desired locations [7], [10], [11]. The modification of the field pattern is done by changing the current distribution in the microcoil array using integrated control electronics. For a simple example, each microcoil can be connected to its own current source for independent magnetic field control as depicted in Fig. 2(b).

Magnetic manipulation of bead-bound cells per se has been widely employed in biology [12]. However, in the conventional approach, a large group of bead-bound cells are statistically pulled all at once using magnetic fields of low spatial resolution, e.g., magnetic fields from magnetic tweezers. In contrast, our approach utilizes microscopic magnetic field patterns generated by the microcoil array circuit, which permits manipulation of many individual cells, moving each cell along a different path.

Because the spatial field pattern can be reconfigured by the IC, our hybrid system offers more flexibility in cell manipulation over the conventional microfluidic system. The conventional microfluidic system moves biological samples in a fixed channel network using predetermined valve controls, and hence, different cell manipulations require different specific microfluidic systems. In contrast, our hybrid system can perform many different types of cell manipulations not by necessarily changing the microfluidic system structure, but by

${ }^{1}$ The magnetic trapping force is given by [7]

$$
\vec{F}=\frac{V \chi}{\mu_{0}} \cdot \vec{\nabla}|B|^{2}
$$

where $V$ and $\chi$ are the volume and magnetic susceptibility of the magnetic bead, respectively, $\mu_{0}$ is the magnetic permeability in vacuum, and $B$ is the magnetic field magnitude.

(a)
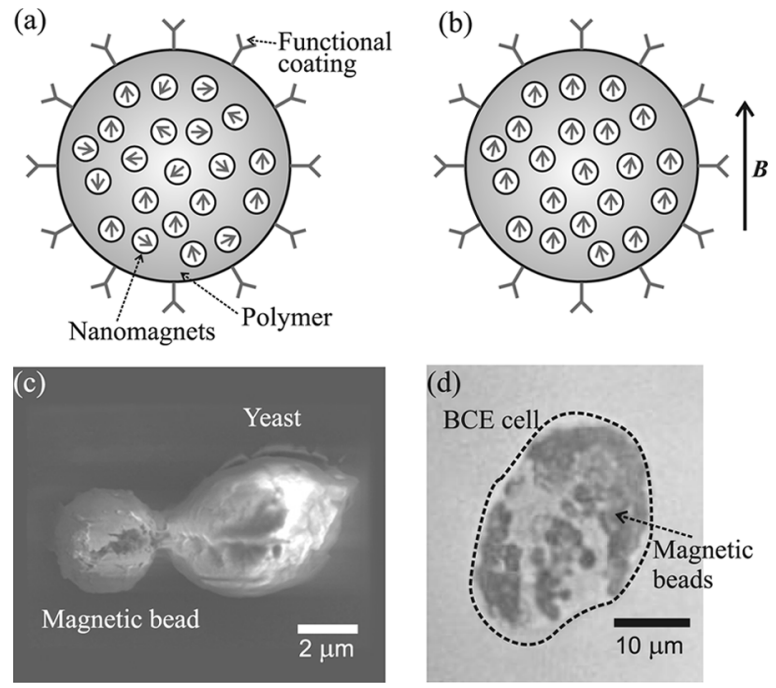

Fig. 3. (a) Inner structure of a magnetic bead with $B=0$. (b) Magnetic bead when $B>0$. (c) Bead-bound yeast cell. (d) BCE cell that contains multiple magnetic beads after natural engulfing processes.

reconfiguring the spatial pattern of the magnetic fields, enabled by the programmability of the IC. In this sense the hybrid system is a programmable microfluidic system.

Our hybrid system may be viewed as a magnetic counterpart (with an enhanced microfluidic system) of the CMOS electric cell manipulation system utilizing dielectrophoresis [6]. Each approach has its own advantages and disadvantages. For example, while the magnetic method requires more sample preparation efforts (magnetic bead attachment), it is more biocompatible as magnetic fields are transparent to cells. Depending on specific experimental needs, a proper choice should be made between the two technologies for optimum manipulation operation.

\section{B. Magnetic Beads and Bead-Bound Cells}

Attaching magnetic beads to cells is a well-established technique from the conventional low-resolution magnetic manipulation approach [12]. As this procedure is essential in our approach as well, we briefly explain the technique here as a background. The magnetic bead is a polymer microsphere containing a large number of nanomagnets as shown in Fig. 3. In the absence of an external magnetic field, the magnetic moments of the nanomagnets are randomly oriented due to thermal agitations [Fig. 3(a)], and hence, the bead has no net magnetic moment. In the presence of an external magnetic field, however, the magnetic moments of the nanomagnets line up overcoming the thermal fluctuations, and the magnetic bead will exhibit an appreciable net magnetic moment as illustrated in Fig. 3(b). It is this magnetic moment that interacts with the magnetic field pattern in the manipulation process.

To attach the magnetic bead to a cell, the surface of the magnetic bead is chemically modified by coating it with specific proteins. This functionalized bead then can be bound to specific target cells. Fig. 3(c) shows an example, a yeast cell attached to a micron-scale magnetic bead. Fig. 3(d) shows another example where multiple magnetic beads of diameter $250 \mathrm{~nm}$ are even engulfed by a bovine capillary endothelial (BCE) cell. Magnetic 

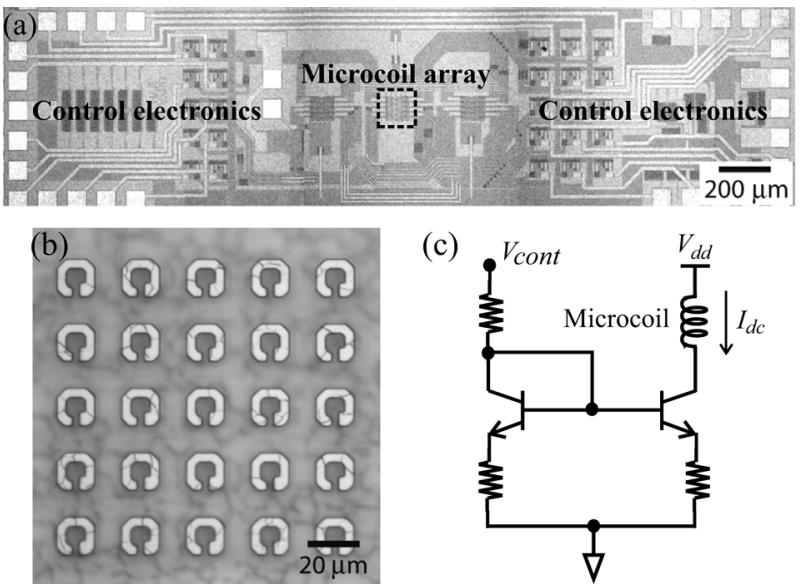

(c)

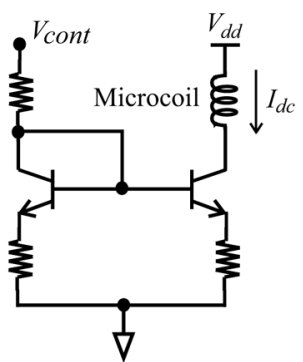

Fig. 4. (a) Micrograph of the SiGe IC $(4 \mathrm{~mm} \times 1 \mathrm{~mm})$. (b) Microcoil array close-up. The outer and inner diameters of each top-metal coil are 14 and $8 \mu \mathrm{m}$, respectively. The center-to-center distance between two adjacent coils is $25 \mu \mathrm{m}$. (c) Control electronics for each microcoil.

beads have been known to be biocompatible: many different types of cells attached to them can grow and live normally [12].

\section{SiGe/Microfluidic Hybrid PROTOTYPE}

This section describes our first hybrid prototype utilizing a SiGe IC, which demonstrates the basic concept with a relatively simple IC operation. This specific prototype was reported in [10], but is included in this paper as a review material for the sake of comprehensiveness.

\section{A. SiGe IC}

Fig. 4(a) is a die micrograph of the SiGe IC. The IC incorporates an array of $5 \times 5$ microcoils [Fig. 4(b)]. Each microcoil is connected to its own current source [Fig. 4(c)] for independent current control. The 3-D structure of the microcoil is as shown in Fig. 2(b). Since the analytical expression of the magnetic field in terms of the coil geometry parameters is very involved, the microcoil is designed and optimized using electromagnetic (EM) field solvers.

An important microcoil design task is to create a single magnetic field magnitude peak at the coil center on the chip surface when an appropriate current is drawn [Fig. 5(a)]. Note in Fig. 5(b) that while the well-designed microcoil produces a single magnetic field magnitude peak on the chip surface, multiple magnetic field magnitude peaks can exist below the surface. An improper design would result in multiple field magnitude peaks on the chip surface, compromising the position control capability. To ensure only one magnitude peak on the chip surface, the microcoil of the SiGe IC is constructed using three coils in different metal layers by connecting them in series with vertical vias [Fig. 2(b)]: the coils in the two lower metal layers are used to shape the magnetic field pattern properly to produce only one field magnitude peak at the coil center on the chip surface.

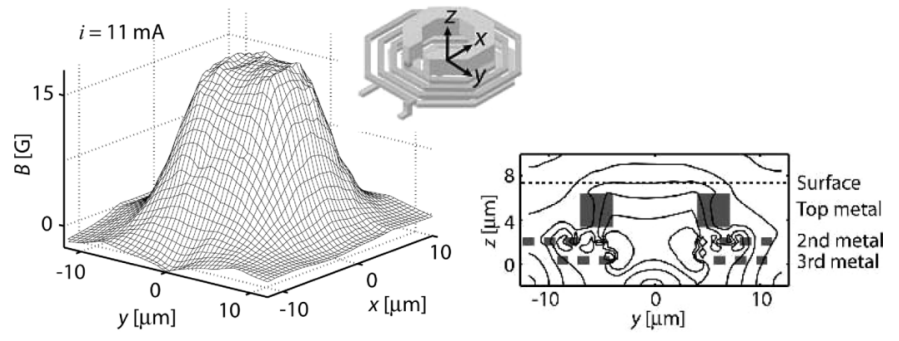

(a)

(b)

Fig. 5. (a) Magnetic field magnitude profile on the surface of the IC calculated for a single microcoil drawing a DC current of $11 \mathrm{~mA}$. (b) Cross section of a microcoil with contours of magnetic field magnitude.

Another important microcoil design goal is to create a magnetic force on the order of tens of piconewtons on the chip surface when a DC current on the order of tens of milliamperes is drawn. Such range of force magnitude is required to transport cells on the chip surface within the microfluid. The magnetic field profile obtained using the EM field solver is used in (1) to calculate the desired magnetic force.

After the microcoil array design, the overall IC is carefully laid out to minimize stray magnetic fields from metal interconnects.

\section{B. SiGe/Microfluidic Hybrid Prototype}

To construct the hybrid system, a microfluidic system is postfabricated on top of the SiGe IC. Fig. 6 shows the sequence of the post-fabrication steps. First, the IC is diced from the wafer [Fig. 6(a)], where the diced area is larger than the designed IC area. This extra area is used as a platform on which a microfluidic system is fabricated. ${ }^{2}$ Second, polyimide is spin-coated and patterned on the surface of the SiGe die to form sidewalls of a microfluidic channel [Fig. 6(b)]. The channel height and width are 30 and $1000 \mu \mathrm{m}$, respectively. Third, a glass coverslip is sealed on top of the channel sidewalls [Fig. 6(c)] using a negative photoresist as a sealing agent. Finally, fluidic tube fittings (inner diameter $\sim 2 \mathrm{~mm}$ ) are separately fabricated in house by casting plastics and are glued to the inlet and outlet of the microfluidic system [Fig. 6(d)].

Fig. 7 is the photo of the completed SiGe/microfluidic hybrid prototype, which sits on top of an in-house fabricated silicon substrate that contains electrical leads. This substrate facilitates handling and electrical interconnections. To prevent electromigration of the chip and to keep the system temperature at biocompatible $37{ }^{\circ} \mathrm{C}$, the overall system of Fig. 7 is mounted on a copper stage cooled by a thermoelectric cooler during the system operation.

\section{Experimental Results}

To demonstrate the manipulation capability of the SiGe/microfluidic hybrid system, magnetic beads (not attached to cells

${ }^{2}$ This specific fabrication method requires a large area in a wafer. This area problem is addressed in the second hybrid prototype construction as discussed in Section IV-E 
(a)

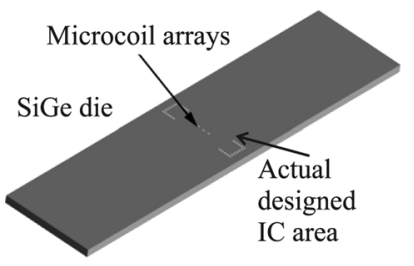

(c)

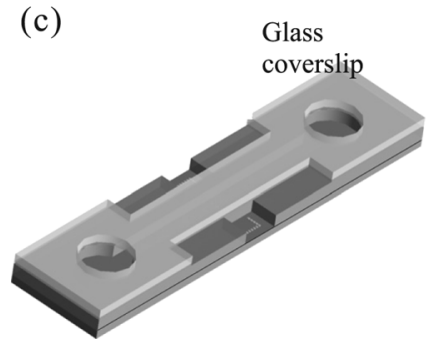

(b)

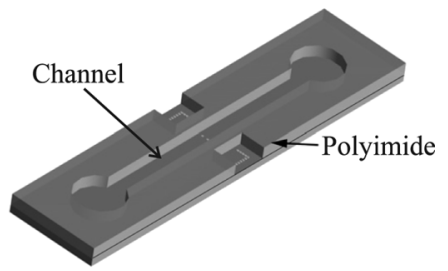

(d)

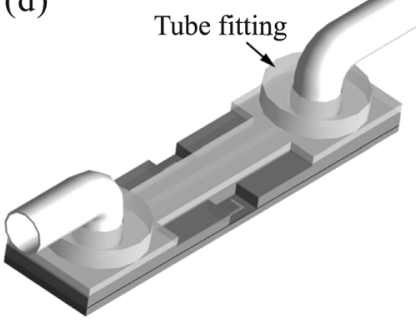

Fig. 6. Post-fabrication of the microfluidic system for the first hybrid prototype.

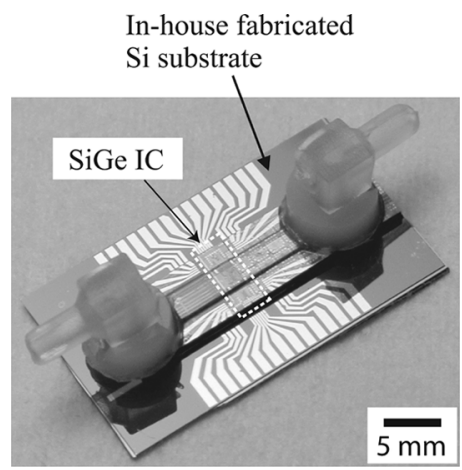

Fig. 7. Photo of the first hybrid prototype.

yet) are used first as target samples. Magnetic beads ${ }^{3}$ of diameter $8.5 \mu \mathrm{m}$ and magnetic susceptibility 0.2 are suspended in distilled water in the microfluidic channel. The bead size is chosen as such that it is comparable to the coil's inner diameter in order to ensure the trapping of a single bead by each coil.

The sequence of the micrographs in Fig. 8 shows the manipulation of individual magnetic beads using the hybrid system. By activating a microcoil with a current of $11 \mathrm{~mA}$, a magnetic field magnitude peak $(B=15 \mathrm{G})$ is obtained at the coil center, at which a single magnetic bead is trapped [Fig. 8(a)]. The average trapping force is approximately $20 \mathrm{pN}$. By deactivating the microcoil and simultaneously activating a coil right below, the bead is moved to the adjacent coil with an average speed of $1 \mu \mathrm{m} / \mathrm{s}$ [Fig. 8(b) and (c)]. While the magnetic bead is trapped, another magnetic bead is trapped by creating an additional field magnitude peak within the coil array [Fig. 8(d)-(f)]. Repeating the same protocol, a larger number of beads can be manipulated independently and simultaneously.

In the second experiment with the $\mathrm{SiGe} /$ microfluidic hybrid prototype, real biological cells (bovine capillary endothelial, or BCE cells) that have engulfed multiple magnetic beads

${ }^{3}$ UMC4F-6548 from Bangs Laboratories, Fishers, IN.
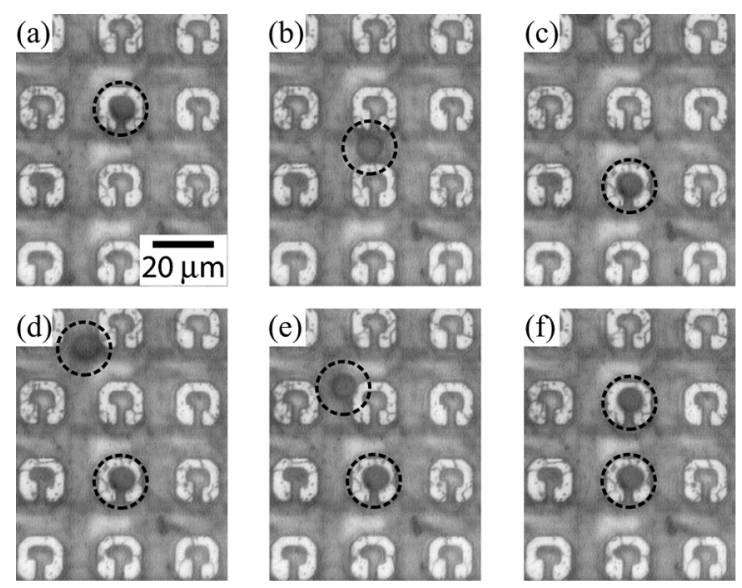

Fig. 8. Manipulation of individual beads with the first prototype.
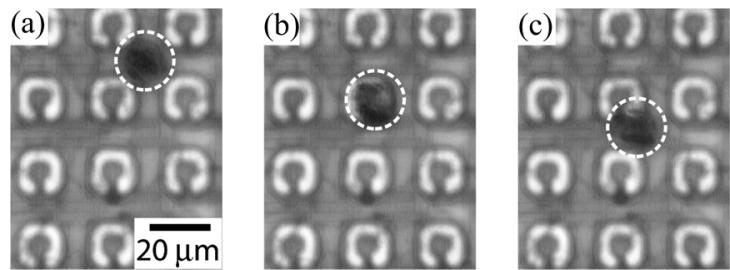

(d) $[\mathrm{C}] \mathrm{Qd}$
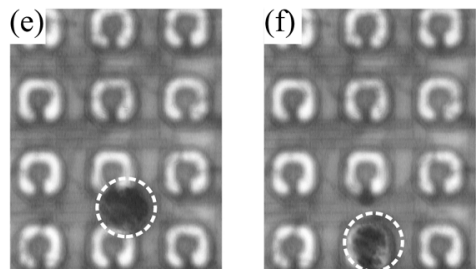

Fig. 9. Manipulation of a BCE cell with the first prototype.

[Fig. 3(d)] are used as target samples. The magnetic beads ${ }^{4}$ used in this experiment have diameters of $250 \mathrm{~nm}$ and magnetite contents of $90 \%$. The submicron bead size is chosen to facilitate engulfment of the magnetic beads by the cells. Before cell manipulation experiments, the chip surface is coated with bovine serum albumin to prevent binding of cells to the chip surface [7].

The sequence of the micrographs in Fig. 9 shows the trapping and translation of a single BCE cell using the hybrid system. A single magnetic field magnitude peak $(B=15 \mathrm{G})$ is created at the center of a microcoil with a current of $11 \mathrm{~mA}$, trapping a single cell with an average force of approximately $50 \mathrm{pN}$ [Fig. 9(a) and (b)].5 Subsequently by moving the field magnitude peak position, the trapped cell is moved to adjacent coils with an average speed of $6 \mu \mathrm{m} / \mathrm{s}$ [Fig. 9(c)-(f)]. Due to the noninvasiveness of the manipulation process and the biocompatible environment maintained by the microfluidic system and the temperature regulation process, the BCE cell remains viable after

\section{${ }^{4}$ PMC-250 from Kisker, Germany.}

${ }^{5}$ While the $11-\mathrm{mA}$ of current is the same as in the previous bead manipulation experiment, the magnetic force of $50 \mathrm{pN}$ in this cell manipulation experiment is bigger than the magnetic force of $20 \mathrm{pN}$ in the previous bead manipulation experiment. This is because the magnetic beads engulfed by the cell in this experiment overall have a larger magnetic susceptibility and occupy a larger volume as compared to the single magnetic bead in the previous bead manipulation experiment. See (1). 


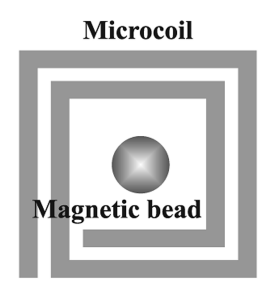

(a) Current on

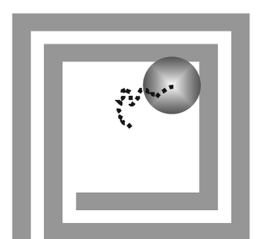

(b) Current off

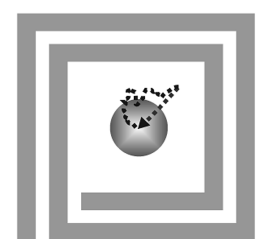

(c) Current on

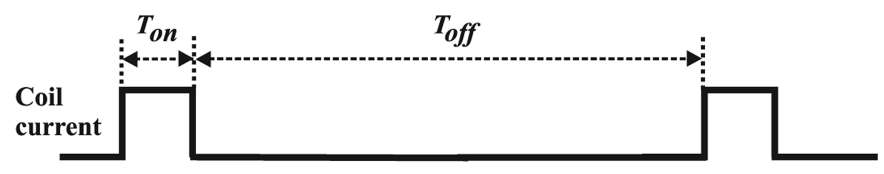

Fig. 10. Basic idea behind the low power operation. (a) Current on; (b) current off; (c) current on.

the experiments, which is demonstrated by successfully reculturing the cells after the experiment.

\section{CMOS/MicROFLUIDIC HYBRID PROTOTYPE}

This section presents our second hybrid prototype, a CMOS/ microfluidic hybrid system. The CMOS IC incorporates digital logic and timing circuits to operate a large microcoil array with low power and high spatial manipulation resolution. This full exploitation of CMOS electronics enables an efficient simultaneous manipulation of many bead-bound cells. The second prototype, with its enhanced capabilities, can be used for various biological applications. For instance, it can assemble a 2-dimensional (2-D) artificial biological tissue at the microscale to study intercellular communications [9]. Important functions/circuits incorporated in the CMOS IC will be presented in this section along with experimental verifications.

\section{A. Low Power Operation of the Microcoil Array}

The simplest protocol to operate an $N \times N$ microcoil array is to implement $N^{2}$ separate on-chip current sources, one for each microcoil, and to continuously flow a current through a microcoil while it is used for transport or trap of a bead-bound cell. For instance, when assembling a biological tissue from $N^{2}$ cells using this protocol, all of the $N^{2}$ microcoils remain on to maintain the trap of the $N^{2}$ cells, one cell for each microcoil. This array operation protocol, which was employed in the first hybrid prototype (Section III), faces a severe power dissipation problem as the number of microcoils $\left(N^{2}\right)$ is increased to handle a large number of cells. A large power dissipation directly translates to thermal failure of the chip and damage to cells through heat shock. To circumvent this problem, the CMOS IC in the second prototype incorporates a low-power protocol to operate a large microcoil array, exploiting the fact that CMOS electronics is much faster than the motion of the cells in fluid. Execution of the protocol is facilitated by digital logic and timing circuits integrated in the CMOS IC.

Using one microcoil, Fig. 10 illustrates the basic idea behind the new protocol. To maintain the trap of a magnetic bead (or a bead-bound cell) within the microcoil, instead of a continuous current flow, the microcoil draws a current for a time duration of $T_{\text {on }}$ and is then deactivated for the next time du- (a)

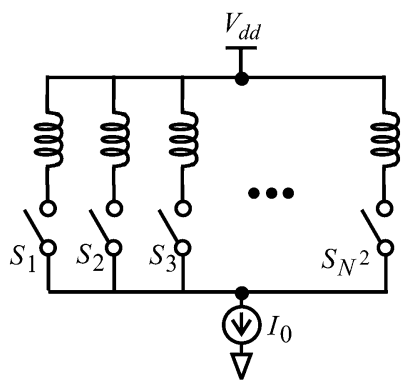

(b)

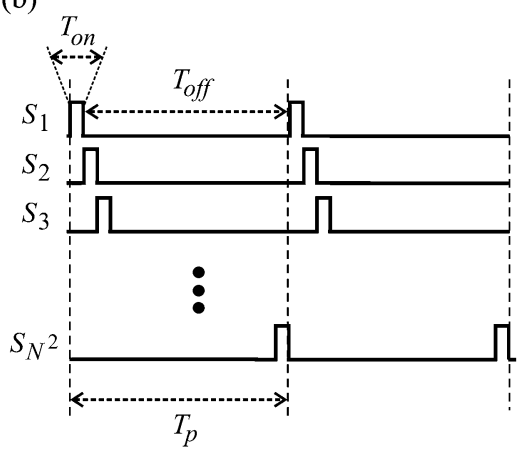

Fig. 11. (a) Simplified schematic of the $N \times N$ microcoil array circuit to perform the sequential current sharing. (b) An example of the sequential current sharing scheme in the case where every microcoil in the $N \times N$ array needs to maintain the trap of a bead-bound cell.

ration of $T_{\text {off }}$. This two-step procedure completed in the period of $T_{p}=T_{\text {on }}+T_{\text {off }}$ is repeated. During the on-time $T_{\text {on }}$, the magnetic bead remains trapped at the center of the microcoil [Fig. 10(a)]. During the following off-time $T_{\text {off }}$, the initially-trapped bead undergoes Brownian motion, walking away from the center of the microcoil [Fig. 10(b)]. However, the microcoil is activated again to repeat the two-step procedure before the bead completely escapes the microcoil, hence trapping the bead back to the center of the microcoil [Fig. 10(c)]. Therefore, with this protocol, the magnetic bead can remain trapped without necessarily supplying a continuous current to the microcoil. Note that $T_{\text {off }}$ needs to be smaller than the characteristic coil escape time due to Brownian motion.

When multiple, $N \times N$ microcoils are used to handle a larger number of bead-bound cells, the two-step procedure for a single microcoil can be sequentially applied to the $N^{2}$ microcoils for low-power operation. This can be done by arranging all the microcoils to share the same current source through switches [Fig. 11(a)] and by sequentially turning on and off a select group of microcoils needed for a specific manipulation by properly clocking the switches. Fig. 11(b) shows an example of this sequential current sharing when all of the $N^{2}$ microcoils need to maintain the trap of $N^{2}$ bead-bound cells, one cell for each microcoil. In a given period $T_{p}=N^{2} \cdot T_{\mathrm{on}}$, each microcoil is activated only once for a duration of $T_{\mathrm{on}}$. At any given moment, there is only one activated microcoil. As long as the off-time of each microcoil, $T_{\text {off }}=\left(N^{2}-1\right) \cdot T_{\text {on }}$, is shorter than the characteristic coil escape time due to Brownian motion, the microcoil array can maintain the trap of the $N^{2}$ cells, sharing the common current source at different times, hence allowing low power operation. Because CMOS electronics is orders of magnitude faster than the cell motions in fluid, the sequential sharing 
of the common current can be executed with high speed to work with a large number of microcoils. Specific design values for $T_{p}$ and $T_{\text {on }}$ are determined from the hydrodynamic properties and number of the cells as well as the size of the microcoils. ${ }^{6}$

The previous example clearly shows the benefit of the sequential current sharing protocol in simultaneous "trap" of multiple bead-bound cells. For simultaneous "transport" of multiple bead-bound cells, while the sequential current sharing scheme still allows a low-power operation, it comes at a price of an increased transport time. This is because bead-bound cells receive force not continuously but for a short duration $\left(T_{\text {on }}\right)$ in each period $\left(T_{p}\right)$. This power-time tradeoff is analogous to principle of work exploited widely in engineering systems. In our case, minimization of power at the expense of the increased transport time is desired to avoid chip failure and to maintain a biocompatible temperature.

\section{B. Enhancement of the Spatial Manipulation Resolution}

By controlling both directions and magnitudes of currents in more than one microcoil, the microcoil array circuit can create the magnetic field magnitude peaks at positions other than the coil centers, effectively enhancing the spatial manipulation resolution.

As an example, Fig. 12 illustrates a case where a magnetic field magnitude peak is created at the midpoint between the two microcoil centers (State 2) along with the two straightforward cases where a magnetic field magnitude peak is generated at either coil center (States 1 and 3). State 2 can be obtained by drawing continuous DC currents of the same magnitude but in opposite directions in the two coils. At the field magnitude peak position in State 2, the field direction is parallel to the chip surface whereas at the field magnitude peak positions in States 1 and 3, the field directions are perpendicular to the chip surface. In State 2, a bead-bound cell is positioned at the midpoint between the two microcoils. This spatial manipulation resolution enhancement scheme is incorporated in the CMOS IC.

In the real implementation of this scheme, the two microcoils in Fig. 12 have to share the same current source because of the sequential current sharing protocol explained in Section IV-A. Therefore, to attain State 2, the common current source is switched back and forth between the two microcoils with the current direction changed in each switching action. The static magnetic field magnitude profile shown in Fig. 12 is still valid in this dynamic case as an average field magnitude that a magnetic bead experiences.

\section{CMOS IC Architecture}

Fig. 13 shows the overall architecture of the CMOS IC that executes both the sequential current sharing protocol and the manipulation resolution enhancement scheme. In this architecture, each microcoil is connected to three FET switches. The two switches labeled $S_{D 1}$ and $S_{D 2}$ are used to change the current direction of the microcoil. The switches labeled $S_{C}$ connect

\footnotetext{
${ }^{6}$ For instance, when the characteristic coil escape time due to the Brownian motion is 10 seconds which is a typical number, $T_{p}$ can be set at 10 seconds while $T_{\text {on }}=10 \mathrm{~ms}$ is sufficient to bring back a bead-bound cell slightly off the coil center back to the coil center, and hence $N=10^{3}$ coils can be operated by sharing the same current source.
}
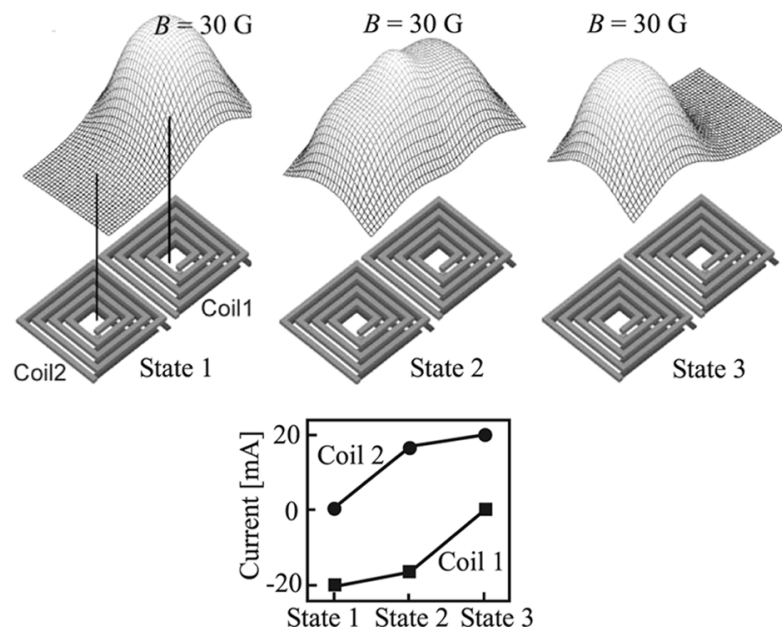

Fig. 12. Enhancement of the spatial manipulation resolution.

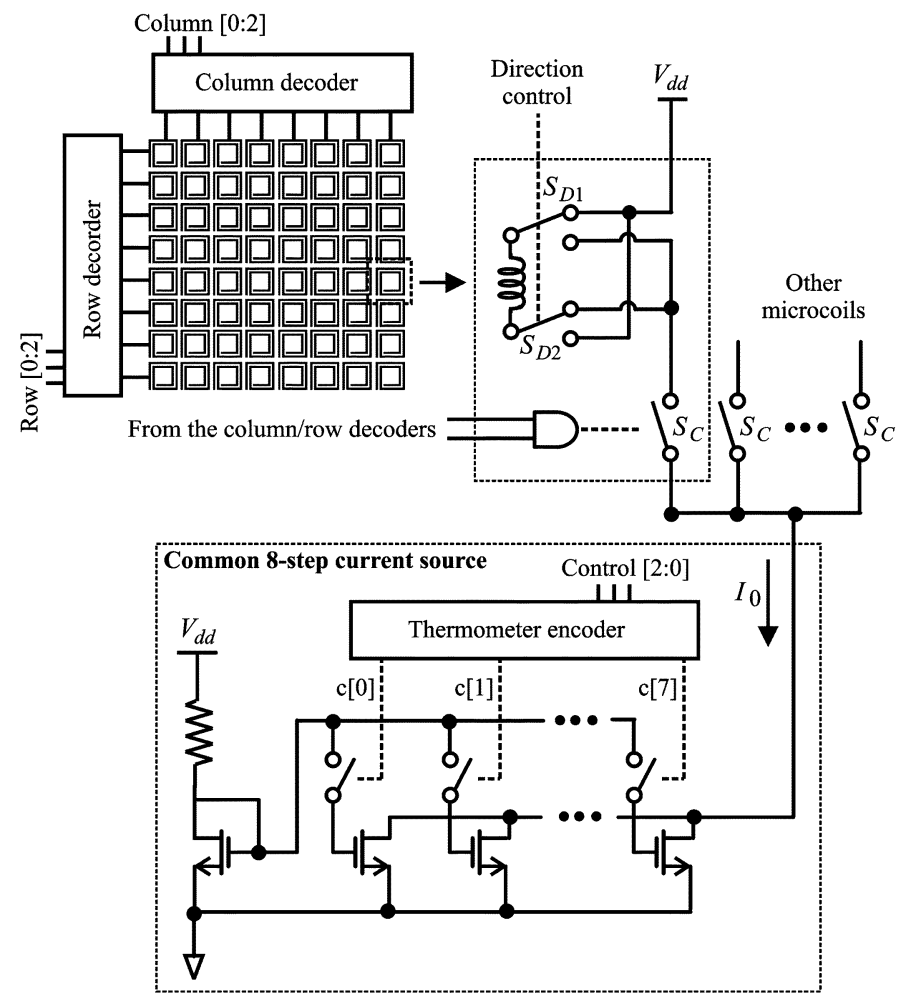

Fig. 13. Overall architecture of the CMOS IC.

the microcoil to the common eight-step current source shared by all the microcoils in the array.

The sequential current sharing is achieved by sequentially connecting and disconnecting a select group of microcoils needed for a specific manipulation to the common eight-step current source. This is done by sending a proper sequence of clock signals to the $S_{C}$ switches using the on-chip column and row decoders.

The resolution enhancement scheme requires controls in both current magnitude and direction. The current magnitude in the microcoil is set by the common eight-step current source in conjunction with the on-chip thermometer encoder. Each branch in the eight-step current source sinks $2.5 \mathrm{~mA}$ and all the eight 
(a)

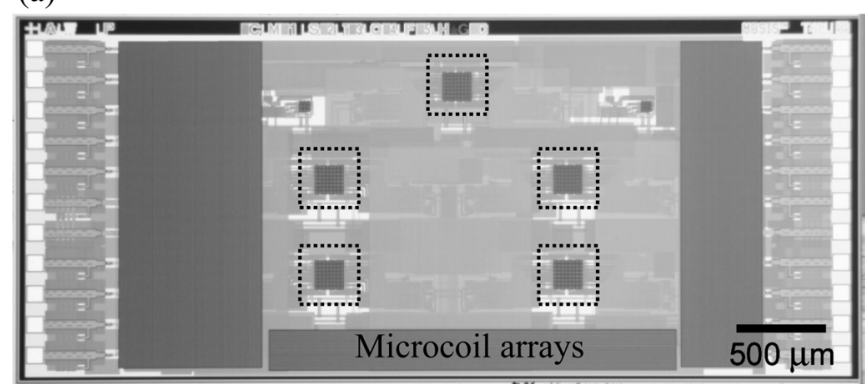

(b)

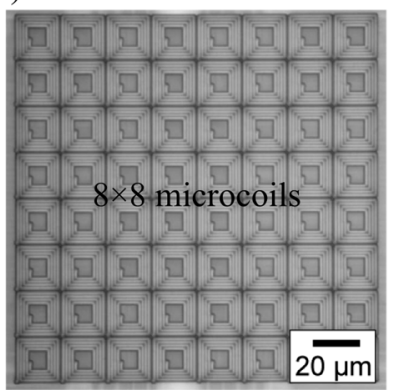

(a)
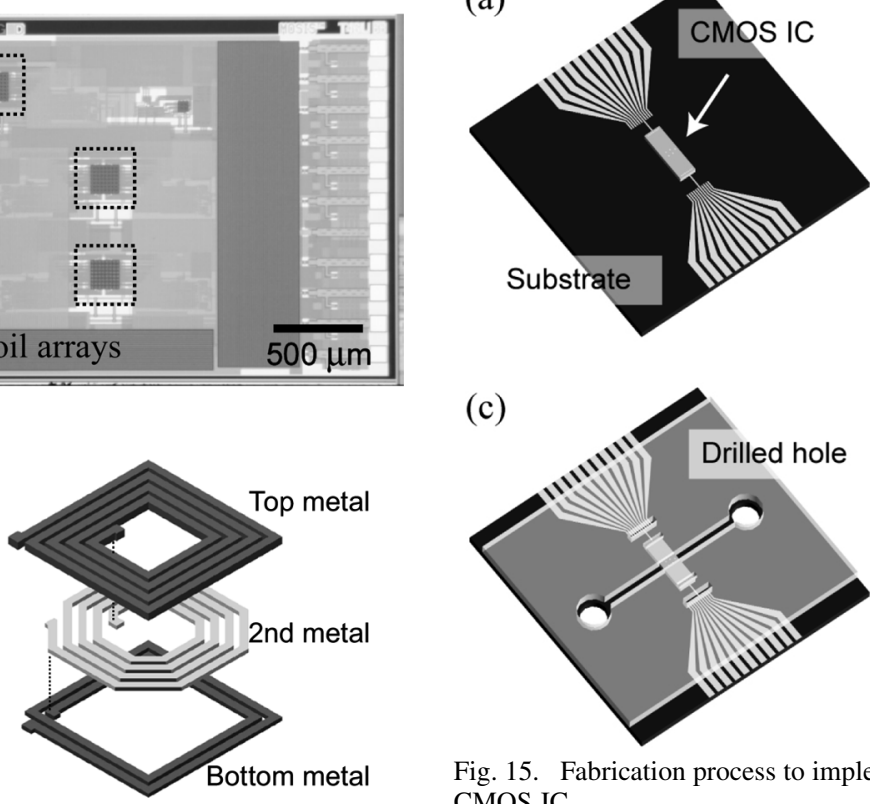

Fig. 14. (a) CMOS IC $(5 \mathrm{~mm} \times 2 \mathrm{~mm})$. (b) The microcoil array close-up and the structure of a microcoil in the array. The outer and inner diameters of the top metal coil are 20 and $10 \mu \mathrm{m}$, respectively. The center-to-center distance between two adjacent coils is $21 \mu \mathrm{m}$.

branches are individually activated by FET switches controlled by the thermometer encoder, achieving eight-step digital control in the magnitude of the current in each microcoil. The microcoil current can reach up to $20 \mathrm{~mA}$ in the increments of $2.5 \mathrm{~mA}$. To choose between two possible current directions in each microcoil, the $S_{D 1}$ and $S_{D 2}$ switches are gated by a current direction control signal. Because only one microcoil is activated at a given time due to the sequential current sharing protocol, a single direction control signal line is shared by all the microcoils. When the common eight-step current source is switched from one microcoil to another microcoil according to the sequential current sharing protocol, the direction control signal flips its polarity if the current direction is to be changed. If the current magnitude is to be changed with the switching, the thermometer encoder will send a command to the eight-step current source to output a proper magnitude of DC current.

\section{CMOS IC Chip}

Fig. 14(a) shows a die micrograph of the CMOS chip fabricated in a $0.18-\mu \mathrm{m}$ technology. The chip contains five $8 \times 8$ microcoil arrays. Fig. 14(b) shows the close-up of one of the arrays along with the structure of a single, identical microcoil in the array. The outer diameter of the microcoil is $20 \mu \mathrm{m}$, and three via-connected metal layers are used to form the microcoil.

\section{E. CMOS/Microfluidic Hybrid Prototype}

To construct the second hybrid prototype, a microfluidic channel is post-fabricated on top of the CMOS IC. Fig. 15 shows the sequence of the post-fabrication steps. First, the CMOS IC chip is glued onto an in-house fabricated silicon substrate that contains lithographically-patterned electrical leads

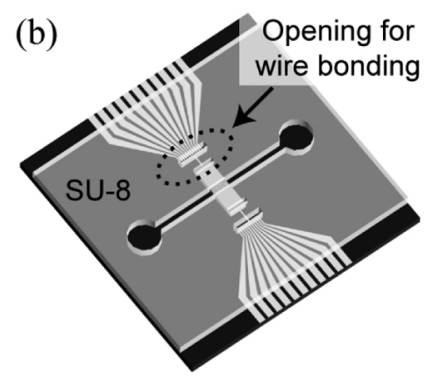

(c)

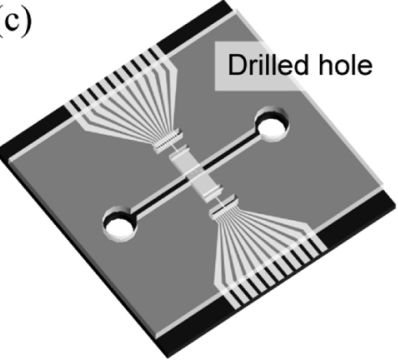

(d)

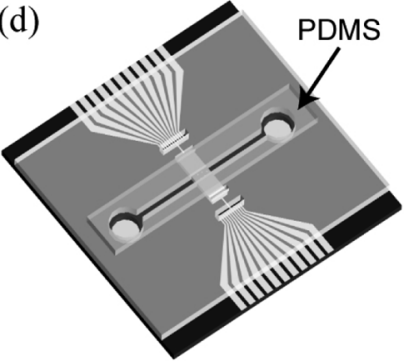

Fig. 15. Fabrication process to implement a microfluidic system on top of the CMOS IC.
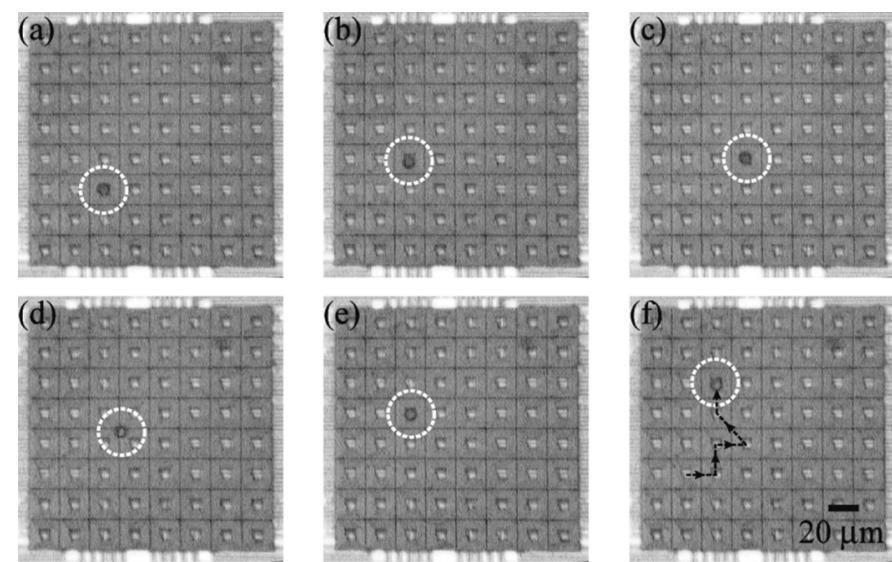

Fig. 16. Manipulation of a single magnetic bead using the CMOS IC/microfluidic hybrid prototype.

[Fig. 15(a)]. The substrate facilitates electrical interconnections and also serves as a platform on which a microfluidic system is fabricated. Second, a negative photoresist (SU-8) layer with thickness $\sim 350 \mu \mathrm{m}$ is spin-coated on the substrate. The SU-8 layer is then lithographically patterned to define the sidewalls of a microfluidic channel and to open up pad areas for wire bonding [Fig. 15(b)]. After patterning the SU-8 structure, two holes are drilled on the substrate to form microfluidic ports [Fig. 15(c)]. A PDMS layer of $\sim 500 \mu \mathrm{m}$ thickness is prepared by cast-coating and cured [13]. The cured PDMS layer cut into a desired shape is placed on top of the SU-8 structure to seal the microfluidic channel.

\section{F. Experimental Results}

Both magnetic beads and BCE cells that have engulfed magnetic beads [Fig. 3(d)] are used to demonstrate the capabilities of the second hybrid prototype.

Fig. 16 shows the manipulation of a single magnetic bead (diameter $8.5 \mu \mathrm{m}$ and magnetic susceptibility 0.2). By activating 

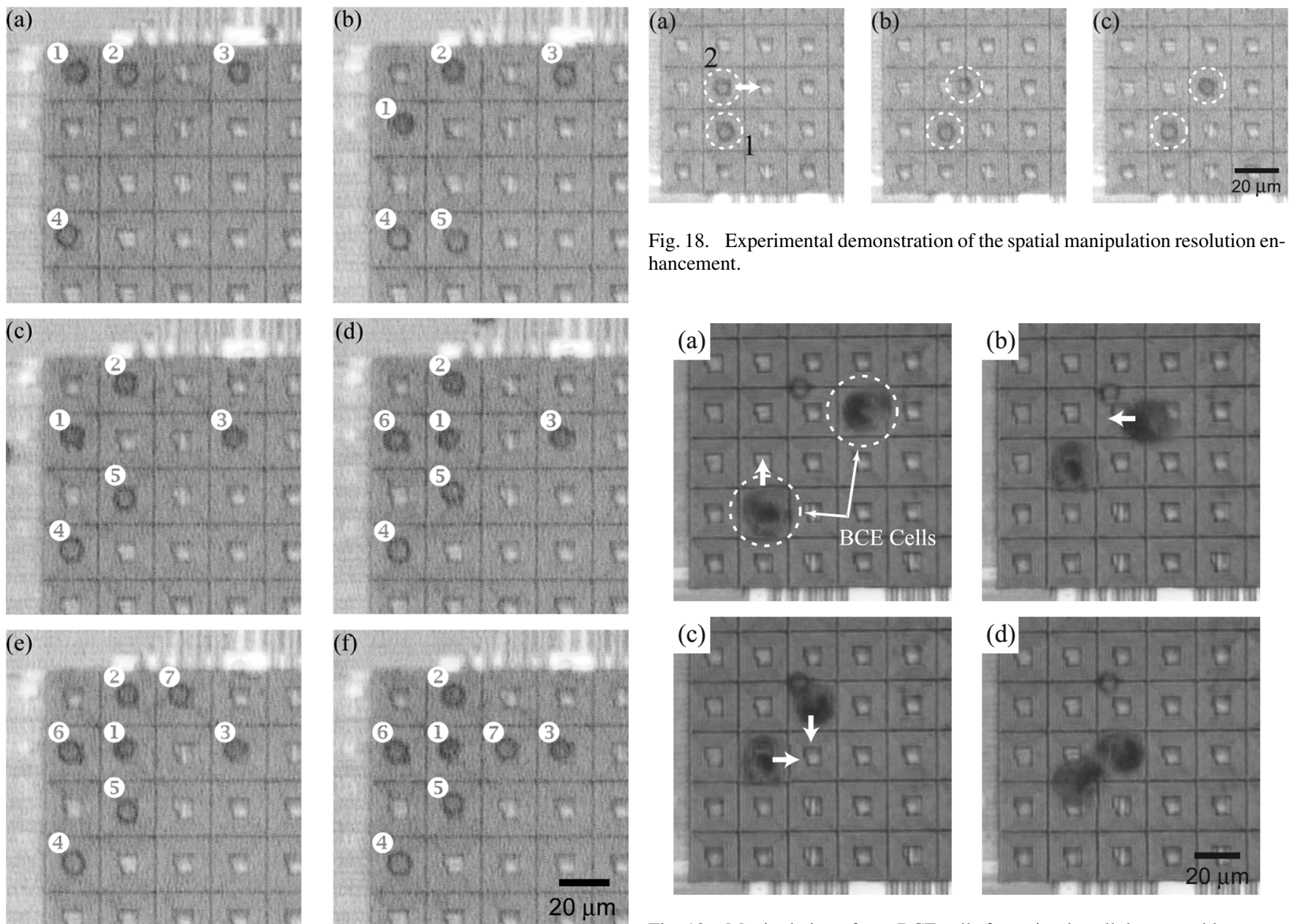

Fig. 18. Experimental demonstration of the spatial manipulation resolution enhancement.
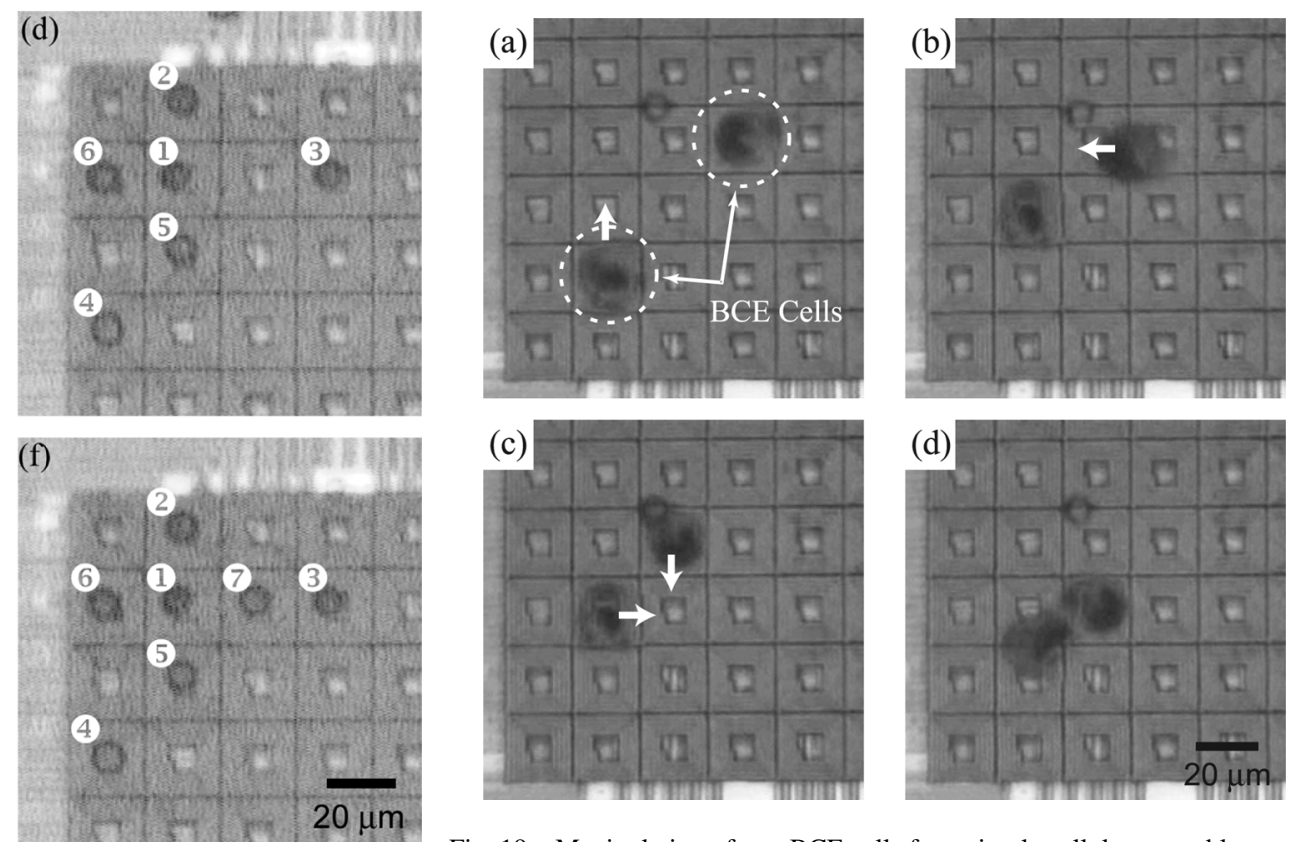

Fig. 19. Manipulation of two BCE cells for a simple cellular assembly.

Fig. 17. Manipulation of multiple magnetic beads using the CMOS IC/microfluidic hybrid prototype.

a microcoil with a DC current of $20 \mathrm{~mA}$, a magnetic field magnitude peak $(B=30 \mathrm{G})$ is produced at the coil center, trapping the bead at the coil center with the average approximate force of $40 \mathrm{pN}$ [Fig. 16(a)]. After the initial trap, the bead is moved to an adjacent microcoil by moving the field magnitude peak [Fig. 16(b)]. Repeating this procedure [Fig. 16(c)-(e)], the bead is transported over a distance of $100 \mu \mathrm{m}$ with the average speed of $11 \mu \mathrm{m} / \mathrm{s}$ following the prescribed path containing vertical, horizontal, and diagonal directions as shown in Fig. 16(f). In this specific experiment, due to the use of a single magnetic bead, the sequential current sharing protocol is not employed.

The low-power sequential current sharing scheme is demonstrated by simultaneously manipulating up to seven magnetic beads as shown in Fig. 17. Every microcoil used in the manipulation is sequentially activated with $T_{\mathrm{on}}=10 \mathrm{~ms}$, while sharing the same DC current of $20 \mathrm{~mA}$ to trap and transport a magnetic bead. Initially four magnetic beads, one bead at one microcoil, are trapped [Fig. 17(a)] by generating four magnetic field magnitude peaks $[B=30 \mathrm{G}]$. While rearranging the four beads, another magnetic bead is trapped and moved [Fig. 17(b) and (c)]. This process is repeated to eventually arrange six magnetic beads in a cross shape [Fig. 17(d)-(f)]. This experiment clearly verifies the low-power operation to simultaneously manipulate individual magnetic beads. verified with the experiment shown in Fig. 18. While one magnetic bead (Bead 1) remains trapped in one microcoil throughout in one microcoil center [Fig. 18(a)], then is positioned and held in the microcoil edge [Fig. 18(b)], and finally is moved to the center of the neighboring microcoil [Fig. 18(c)]. The sequential current sharing protocol is being employed at the same time for low-power operation.

This second prototype may be utilized for various biological applications, for instance, a microscale artificial tissue assembly. The experiment shown in Fig. 19 with two BCE cells (that have engulfed multiple magnetic beads [Fig. 3(d)] whose diameter is $250 \mathrm{~nm}$ ) demonstrates the feasibility for such application. Initially the two cells are trapped in two separate microcoils [Fig. 19(a)]. They are then transported simultaneously [Fig. 19(b) and (c)] toward each other, and are eventually joined [Fig. 19(d)] by holding them together.

\section{CONCLUSION}

In this paper we introduced an IC/microfluidic hybrid system as a new biolab-on-IC. The programmability and speed of the IC allows efficient, precise, and versatile manipulation of many individual magnetic beads and bead-bound cells suspended within
The enhancement of the spatial manipulation resolution is the experiment, another magnetic bead (Bead 2) is first trapped 
the microfluidic system fabricated on top of the IC. The microfluidic system provides a biocompatible environment. The hybrid system is a miniaturized, self-contained system that integrates control electronics. Due to the low fabrication cost, the hybrid system can be used as a single-use, disposable device.

The IC/microfluidic hybrid system offers an interesting direction in the field of biolab-on-IC with the prospect of enabling important biological applications. One significant potential application is to assemble a 2-D biological tissue at the microscale. By bringing cells one by one with precise spatial control, the hybrid system can build an artificial tissue in a standardized and repeatable manner with tight demographic quality control measures. The assembled tissue can be used as a model tissue to study communications between different types of cells or to test drug efficacy.

There still remain IC design challenges to further mature the hybrid technology. An important task is to perfect the long-term biocompatibility by incorporating an on-chip temperature regulator to keep the system temperature below $37^{\circ} \mathrm{C}$ (in the reported prototypes, the temperature regulation is performed through an off-chip thermoelectric cooler). Another important task is to develop an all-electrical on-chip sensor (for each microcoil) that can map the distribution of bead-bound cells without relying on optics. Since the magnetic bead changes the resonance characteristics of the microcoil due to the bead's magnetic flux, a radio-frequency (RF) signal may be used for the sensing purpose [7]. Such RF sensors will render bulky optics unnecessary, realizing a true biolab-on-IC.

\section{ACKNOWLEDGMENT}

The authors would like to thank L. DeVito and S. Feindt of Analog Devices Inc. and B. Park of Samsung Electronics for their continued support and chip fabrications. The authors also thank E. Nam of Electronics and Telecommunication Research Institute (ETRI), Korea, for support through foundry service and gift. The authors acknowledge K. Parker, D. Weitz, and W. Andress of Harvard for invaluable discussions. E. Alsberg and D. Ingber of Harvard Medical School and Children's Hospital (Boston) provided the bovine capillary endothelial cells. AnSoft, Sonnet, and Agilent donated their field solvers.

\section{REFERENCES}

[1] R. A. Kaul, N. I. Syed, and P. Fromherz, "Neuron-semiconductor chip with chemical synapse between identified neurons," Phys. Rev. Lett., vol. 92, no. 3, pp. 38102/1-38102/4, Jan. 2004.

[2] P. Fromherz, "Joining ionics and electronics: semiconductor chips with ion channels, nerve cells, and brain tissue," in IEEE ISSCC Dig. Tech. Papers, Feb. 2005, pp. 76-77.

[3] A. Hassibi and T. H. Lee, "A programmable electrochemical biosensor array in $0.18 \mu \mathrm{m}$ standard CMOS," in IEEE ISSCC Dig. Tech. Papers, Feb. 2005, pp. 564-565.

[4] B. Eversmann, C. Paulus, F. Hofmann, R. Brederlow, B. Holzapfl, P. Fromherz, M. Brenner, M. Schreiter, R. Gabl, K. Plehnert, M. Steinhauser, G. Eckstein, D. Schmitt-Landsiedel, and R. Thewes, "A $128 \times$ 128 bio-sensor array for extracellular recording of neural activity," in IEEE ISSCC Dig. Tech. Papers, Feb. 2003, pp. 222-223.

[5] P. Cailat, M. Belleville, F. Clerc, and C. Massit, "Active CMOS biochips: an electro-addressed DNA probe," in IEEE ISSCC Dig. Tech. Papers, Feb. 1998, pp. 272-273.
[6] N. Manaresi, A. Romani, G. Medoro, L. Altomare, A. Leonardi, M. Tartagni, and R. Guerrieri, "A CMOS chip for individual cell manipulation and detection," IEEE J. Solid-State Circuits, vol. 38, no. 12, pp. 2297-2305, Dec. 2003.

[7] H. Lee, "Microelectronic/microfluidic hybrid system for the manipulation of biological cells," Ph.D. dissertation, Harvard Univ., Cambridge, MA, 2005.

[8] P. J. Lee, P. J. Hung, R. Shaw, L. Jan, and L. P. Lee, "Microfluidic application integrated device for monitoring direct cell-cell communication via gap junctions between individual cell pairs," Appl. Phys. Lett., vol. 86, pp. 223902/1-223902/3, 2005.

[9] S. N. Bhatia and C. S. Chen, "Tissue engineering at the microscale," Biomed. Microdevices, vol. 2, pp. 131-144, 1999.

[10] H. Lee, Y. Liu, E. Alsberg, D. E. Ingber, R. M. Westervelt, and D. Ham, "An IC/microfluidic hybrid microsystem for 2-D magnetic manipulation of individual biological cells," in IEEE ISSCC Dig. Tech. Papers, Feb. 2005, pp. 80-81.

[11] H. Lee, A. M. Purdon, and R. M. Westervelt, "Manipulation of biological cells using a microelectromagnet matrix," Appl. Phys. Lett., vol. 85, pp. 1063-1065, 2004.

[12] U. Häfeli, Scientific and Clinical Applications of Magnetic Carriers. New York: Plenum Press, 1997.

[13] G. M. Whitesides, E. Ostuni, S. Takayama, X. Jiang, and D. E. Ingber, "Soft lithography in biology and biochemistry," Annu. Rev. Biomed. Eng., vol. 3, pp. 335-373, 2001.

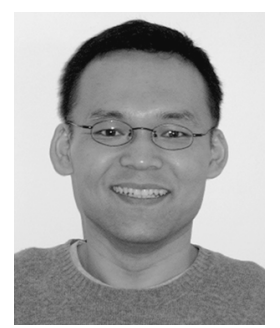

Hakho Lee received the B.S. degree in physics from Seoul National University, Seoul, Korea, in 1998, and the Ph.D. degree in physics from Harvard University, Cambridge, MA, in 2005. For the Ph.D., he developed a new type of micro-total-analysis-systems $(\mu \mathrm{TAS})$ for biological experiments based on integrated circuits and microfluidics.

In 2005, he joined the Center for Molecular Imaging Research at the Massachusetts General Hospital and Harvard Medical School as a Research Associate. His current research interests include microelectronic actuators for biological cell manipulation, and magnetic and optical biosensors for medical imaging.

Dr. Lee ranked first in the nationwide college entrance in 1992, and won the grand prize in natural sciences at the nationwide Undergraduate Thesis Competition, Korea, in 1998. He was also a recipient of the Harvard Russell Fellowship.

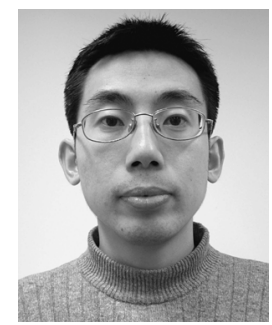

Yong Liu (S'03) received the B.S.E.E. and M.S.E.E. degrees from Tsinghua University, Beijing, China, in 2000 and 2003, respectively. He is currently working toward the Ph.D. degree in electrical engineering at Harvard University, Cambridge, MA.

In 2001, he was with Tsinghua Tongfang Microelectronics Company, Beijing, working on the second-generation Chinese RF ID card. During the summer of 2005, he was with the Mixed-signal Communications IC Design Group at the IBM T. J. Watson Research Center, Yorktown Heights, NY, where he designed a high-performance autonomic PLL for high-speed data links. His current research interests include biological laboratories on an integrated circuit and RF and mixed-signal integrated circuits for communications.

Mr. Liu received the Seagate Scholarship for Extraordinary Freshman and the Motorola Scholarship at Tsinghua University in 1996 and 2001, respectively. He was the recipient of the 2nd Prize in the National Graduate EDA Competition, China, in 2002, and the winner of the Analog Devices Outstanding Student Designer Award in 2004 


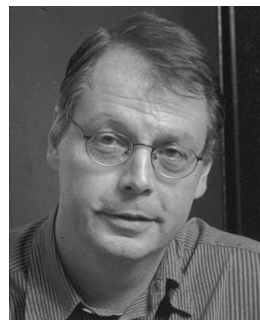

Robert M. Westervelt received the Ph.D. degree in physics from the University of California, Berkeley, in 1977. Following a postdoctoral appointment at Berkeley, he moved to Harvard University, Cambridge, MA, where he is currently a Mallinckrodt Professor of Applied Physics and Physics.

His group investigates the quantum behavior of electrons inside nanoscale semiconductor structures and develops tools for the manipulation of biological systems. Current research topics include: imaging electron flow through nanoscale devices at low temperatures using scanning probe microscopy, making artificial molecules composed of few-electron quantum dots for quantum information processing, and the development of hybrid integrated circuit/microfluidic chips to trap, move, assemble, and sort biological cells and small particles in fluids. He is Director of the NSF-funded Nanoscale Science and Engineering Center at Harvard University, which includes participants at the Massachusetts Institute of Technology, the University of California, Santa Barbara and the Museum of Science, Boston. Previously Westervelt was Director of the Materials Research Science and Engineering Center, and Co-Director of the Joint Services Electronics Program at Harvard.

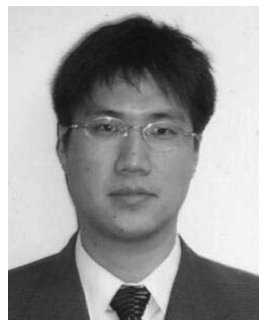

Donhee Ham (M'02) received the B.S. degree in physics from Seoul National University, Seoul, Korea, in 1996, and the Ph.D. degree in electrical engineering from the California Institute of Technology (Caltech), Pasadena, in 2002.

From 1997 to 1998, he was with the Laser Interferometer Gravitational Wave Observatory (LIGO), Pasadena. In 2000, he was with the IBM T. J. Watson Research Center, Yorktown Heights, NY. In 2002, he joined the Faculty of the Division of Engineering and Applied Sciences, Harvard University, Cambridge, MA, as an Assistant Professor of electrical engineering. His research is focused on ultrafast solid-state electronics, specifically, RF and microwave integrated circuits, soliton and nonlinear wave electronics, and nanoscale quantum-effect devices for gigahertz and terahertz circuits. His research also examines biological laboratories on an integrated circuit.

Dr. Ham ranked first in the School of Natural Science, Seoul National University upon his graduation and received the Presidential Top Honor Prize. He was a recipient of the Caltech Li Ming Scholarship, an IBM Graduate Fellowship, and the 2002 Caltech Charles Wilts Doctoral Thesis Prize for outstanding $\mathrm{Ph} . \mathrm{D}$. research in electrical engineering. He was also the recipient of the 2003 IBM Faculty Partnership Award. 